\title{
CCR1 Activation Promotes Neuroinflammation Through CCR1/TPR1/ERK1/2 Signaling Pathway After Intracerebral Hemorrhage in Mice
}

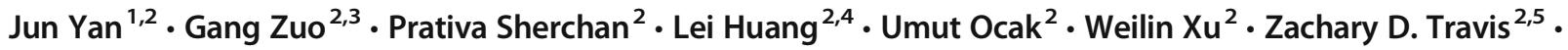 \\ Wenna Wang ${ }^{2}$. John H. Zhang ${ }^{2,4,6}$. Jiping Tang ${ }^{2}$ (1)
}

Published online: 2 January 2020

(C) The American Society for Experimental NeuroTherapeutics, Inc. 2020

\begin{abstract}
The activation of C-C chemokine receptor type 1 (CCR1) has been shown to be pro-inflammatory in several animal models of neurological diseases. The objective of this study was to investigate the activation of CCR1 on neuroinflammation in a mouse model of intracerebral hemorrhage (ICH) and the mechanism of CCR1/tetratricopeptide repeat 1 (TPR1)/extracellular signalregulated kinase 1/2 (ERK1/2) signaling pathway in CCR1-mediated neuroinflammation. Adult male CD1 mice $(n=210)$ were used in the study. The selective CCR1 antagonist Met-RANTES was administered intranasally at $1 \mathrm{~h}$ after autologous blood injection. To elucidate potential mechanism, a specific ERK1/2 activator (ceramide C6) was administered prior to Met-RANTES treatment; CCR1 activator (recombinant CCL5, rCCL5) and TPR1 CRISPR were administered in naïve mouse. Neurobehavioral assessments, brain water content, immunofluorescence staining, and western blot were performed. The endogenous expressions of CCR1, CCL5, TPR1, and p-ERK1/2 were increased in the brain after ICH. CCR1 were expressed on microglia, neurons, and astrocytes. The inhibition of CCR1 with Met-RANTES improved neurologic function, decreased brain edema, and suppressed microglia/macrophage activations and neutrophil infiltration after ICH. Met-RANTES treatment decreased expressions of CCR1, TPR1, p-ERK, TNF- $\alpha$, and IL-1 $\beta$, which was reversed by ceramide C6. The brain CCR1 activation by rCCL5 injection in naïve mouse resulted in neurological deficits and increased expressions of CCR1, TPR1, p-ERK, TNF- $\alpha$, and IL-1 $\beta$. These detrimental effects of rCCL5 were reversed by TPR1 knockdown using TPR1 CRISPR. Our study demonstrated that CCR 1 activation promoted neuroinflammation through CCR1/TPR1/ERK1/2 signaling pathway after ICH in mice. CCR1 inhibition with Met-RANTES attenuated neuroinflammation, thereby reducing brain edema and improving neurobehavioral functions. Targeting CCR1 activation may provide a promising therapeutic approach in the management of ICH patients.
\end{abstract}

Key Words Intracerebral hemorrhage $\cdot \mathrm{C}-\mathrm{C}$ chemokine receptor type $1 \cdot$ Met-RANTES $\cdot$ brain edema $\cdot$ neuroinflammation

Jun Yan and Gang Zuo contributed equally to this work.

Electronic supplementary material The online version of this article (https://doi.org/10.1007/s13311-019-00821-5) contains supplementary material, which is available to authorized users.

Jiping Tang

jtang@1lu.edu

1 Department of Neurosurgery, Guangxi Medical University Cancer Hospital, Nanning 530021, Guangxi, China

2 Department of Physiology and Pharmacology, School of Medicine, Loma Linda University, 11041 Campus Street, Loma Linda, CA 92354, USA
3 Department of Neurosurgery, The Affiliated Taicang Hospital, Soochow University, Taicang, Suzhou 215400, Jiangsu, China

4 Department of Neurosurgery, School of Medicine, Loma Linda University, Loma Linda, CA 92354, USA

5 Department of Earth and Biological Sciences, Loma Linda University, Loma Linda, CA 92350, USA

6 Department of Anesthesiology, School of Medicine, Loma Linda University, Loma Linda, CA 92354, USA 


\section{Background}

Spontaneous intracerebral hemorrhage (ICH) is a severe fatal stroke subtype that accounts for 10 to $15 \%$ of all stroke patients, which associated with high mortality, morbidity, and high socioeconomic burden $[1,2]$. The deposited blood in brain causes local tissue deformation and its expansion is regarded as the primary brain injury to damage the brain tissue. Red blood cell debris and blood products initiate secondary brain injury including the inflammatory response after ICH. The brain resident microglia cells are prominently involved in this response and become activated within minutes after ICH. These series of events can further lead to tissue damage, blood-brain barrier disruption, and brain edema [3-5]. Therefore, anti-inflammatory strategies may have potential therapeutic effects against ICH and improve neurological outcomes after ICH [6-9].

Chemokines are a family of low molecular weight proteins with an essential role in leukocyte trafficking during inflammation. The $\mathrm{C}-\mathrm{C}$ chemokine receptor type 1(CCR1) encodes a member of beta chemokine receptor family, which belongs to $\mathrm{G}$ protein-coupled receptors (ref). In brain, several studies have observed the expression of CCR1 on neurons, microglia, and astrocytes [10-12]. Following neonatal hypoxic ischemia, CCR1 was predominantly expressed in the rat cortex, thalamus, striatum, hippocampus, cerebral endothelium, and ependyma [11]. The binding of CCR1 to its endogenous ligand, RANTES (CCL5), contributed to inflammatory process in Alzheimer's disease, autoimmune encephalomyelitis (EAE), and cerebral ischemia [10, 13, 14]. Met-RANTES, an N-terminally modified human RANTES, selectively inhibits CCR1 activity [15]. Met-RANTES showed the antiinflammatory efficacy in the animal models of acute and chronic inflammation $[16,17]$. However, the potential role of CCR1 activation in neuroinflammation and underlying mechanism as well as the treatment effect of Met-RANTES have not been investigated in the setting of ICH.

Tetratricopeptide repeat 1 (TPR1) is a ubiquitous protein with three TPR motifs [18]. In HEK293 cells, the activation of TPR1/ERK1/2 signaling pathway upregulated proinflammatory mediators including TNF- $\alpha$, IL- 6 , and IL- $1 \beta$ $[19,20]$. Recent study showed that CCR1 may link G $\alpha 14 / 16$ to stimulate TPR1/Ras/MEK/ERK pathway [19-21]. In addition, CCL5/CCR1 axis could activate ERK and AKT kinases in human adipose tissue-derived stromal cells [22].

In the present study, we hypothesize that the CCR1 activation promotes neuroinflammation partially through CCR1/TPR1/ERK1/2 signaling pathway in a mouse model of ICH. The CCR1 inhibition with Met-RANTES would attenuate neuroinflammation and improve neurological outcomes in ICH mice.

\section{Methods}

\section{Animals}

A total of 210 8-week-old male CD1 mice (weight about 30 $40 \mathrm{~g}$, Charles River, MA, USA) were used. Mice were housed in a 12-h light/dark cycle at a temperature and humidity controlled room for a minimum of 3 days before surgery, with free access to food and water. All the experimental protocols and procedures for this study were approved by the Institutional Animal Care and Use Committee (IACUC) at Loma Linda University in accordance with the National Institutes of Health's Guide for the Care and Use of Laboratory Animals. The manuscript adheres to the ARRIVE (Animal Research: Reporting of In Vivo Experiments) guidelines for reporting animal experiments.

\section{Experiment Design}

In the present study, all mice were randomly assigned to the following experiments. The experimental design was shown in Figure S1 (Additional file 1). The summary of experimental groups, animal numbers, and mortality rate in the study was listed in Table S1 (Additional file 2).

All the experimental groups' information was blinded to the researchers who performed the surgery/neurobehavioral tests and data analysis. Samples' size of each experiment was determined according to previous research using

\section{- Experiment 1}

To evaluate time course of endogenous CCR1, CCL5, TPR1, and p-ERK1/2 expressions in brain after ICH, a total of 36 mice were randomly divided into 6 groups $(n=6 /$ group): sham, ICH after 3, 6, 12, 24, and $72 \mathrm{~h}$. Western blot analysis was used to detect expressions of CCR1, CCL5, TPR1, and p-ERK1/2 in the ipsilateral/right hemisphere of each group. Additional two mice were used for immunofluorescence staining at $24 \mathrm{~h}$ post-ICH. The cellular localization of CCR1 was evaluated using double-labeling immunofluorescence staining to co-localize CCR1 with ionized calcium binding adaptor molecule 1 (Iba-1), neuronal specific nuclear protein $(\mathrm{NeuN})$, and glial fibrillary acidic protein (GFAP) at $24 \mathrm{~h}$ after ICH.

\section{- Experiment 2}

To evaluate the anti-inflammatory effect of CCR1 inhibition with the selective antagonist Met-RANTES in ICH, a total of 30 mice were randomly divided into 5 groups $(n=6 /$ group): sham, ICH + vehicle (PBS), ICH + Met-RANTES $(0.15 \mu \mathrm{g} / \mathrm{kg}), \mathrm{ICH}+$ Met-RANTES $(0.5 \mu \mathrm{g} / \mathrm{kg}), \mathrm{ICH}+$ MetRANTES $(1.5 \mu \mathrm{g} / \mathrm{kg})$ [23]. Met-RANTES were administered 
intranasally at $1 \mathrm{~h}$ post-ICH. Neurobehavioral tests and brain water content (BWC) were assessed at $24 \mathrm{~h}$ post-ICH. Based on the 24-h outcomes, the optimal dose of $0.5 \mu \mathrm{g} / \mathrm{kg}$ was chosen for the rest of experiments involving in MetRANTES treatment. For neurobehavioral tests and BWC at $72 \mathrm{~h}$ post- $\mathrm{ICH}$, a total of 18 mice were randomly divided into 3 groups ( $n=6 /$ group): sham, ICH + vehicle (PBS) and ICH + Met-RANTES $(0.5 \mu \mathrm{g} / \mathrm{kg})$. To evaluate the effects of CCR1 inhibition with Met-RANTES on neuroinflamation at $24 \mathrm{~h}$ after ICH, a total of 30 mice were randomized into 3 groups ( $n=10 /$ group): sham, ICH + vehicle (PBS) and ICH + Met-RANTES $(0.5 \mu \mathrm{g} / \mathrm{kg})$. Immunofluorescence staining ( $n=4 /$ group) of Iba- 1 , IL- $1 \beta$, and myeloperoxidase (MPO) was quantified by counting Iba-1, IL- $1 \beta$, and MPOpositive cells in the perihematomal area $(20 \times$, averaged from 3 field view/slice, 10 slices/mouse). Western blot ( $n=6 /$ group) was also performed to quantifying the protein levels of Iba-1, IL-1 $\beta$, and MPO.

\section{- Experiment 3}

To assess long-term neurobehavioral function, a total of 24 mice were randomized into 3 groups ( $n=8 /$ group): sham, $\mathrm{ICH}+$ vehicle (PBS), ICH + Met-RANTES $(0.5 \mu \mathrm{g} / \mathrm{kg})$. Long-term neurological evaluation was performed as follows: The foot fault test and rotarod test were performed on days 7, 14 , and 21 post-ICH. Morris water maze test was conducted on days $21-25$ post-ICH.

\section{- Experiment 4}

To elucidate ERK1/2 signaling pathway in CCR1mediated neuroinflammation after ICH, a total of 30 mice were randomly divided into five groups ( $n=6 /$ group): sham, ICH + vehicle (PBS), ICH + Met-RANTES $(0.5 \mu \mathrm{g} / \mathrm{kg}), \mathrm{ICH}+$ Met-RANTES + vehicle (DMSO), ICH + Met-RANTES + ceramide C6. CERAMIDE C6, a selective ERK1/2 activator was administered by intracerebroventricular injection $24 \mathrm{~h}$ prior to ICH induction followed by the intranasal delivery of specific CCR 1 antagonist Met-RANTES $(0.5 \mu \mathrm{g} / \mathrm{kg})$ at $1 \mathrm{~h}$ after ICH. Neurobehavioral tests and western blot were performed at $24 \mathrm{~h}$ post-ICH.

\section{- Experiment 5}

To further verify the relationship between CCR1 activation and TPR $1 /$ ERK $1 / 2$ signaling pathway, a total of 30 mice were randomized into five groups ( $n=6 /$ group): Naive, Naive + Vehicle, Naive + rCCL5, Naive + rCCL5 + Scramble CRISPR, Naive + rCCL5 + TPR1 CRISPR. The CCR1 agonist, rCCL5, was administrated in naïve mice by intracerebroventricular injection. TPR1 CRISPR or Scramble CRISPR was intracerebroventricularly injected $24 \mathrm{~h}$ prior to rCCL5 administration. Neurobehavioral tests and western blot were performed at $24 \mathrm{~h}$ after rCCL5 injection.

\section{Mouse Model of Intracerebral Hemorrhage}

ICH was induced by the autologous whole blood injection as previously described [24, 25]. Briefly, mice were anesthetized with ketamine $(100 \mathrm{mg} / \mathrm{kg})$ and xylazine $(10 \mathrm{mg} / \mathrm{kg})(2: 1 \mathrm{v} / \mathrm{v}$, intraperitoneal injection) and positioned prone in a stereotactic head frame (Kopf Instruments, Tujunga, CA, USA). An artificial tears ointment (Rugby, Livonia, MI, USA) was used to keep the eyes moist during surgery. Arterial blood was collected in a nonheparinized capillary tube, transferred immediately into a 27 -gauge needle on a $250-\mu 1$ Hamilton syringe. A 1-mm cranial burr hole was drilled and the Hamilton syringe was inserted stereotactically into the right basal ganglia (coordinates: $0.2 \mathrm{~mm}$ posterior and 2.2 lateral to the bregma, $3.5 \mathrm{~mm}$ below the dura). A total volume of $30 \mu \mathrm{L}$ blood was infused into the right basal ganglia using a microinfusion pump (Stoelting, Harvard Apparatus, Holliston, MA), with $5 \mu \mathrm{L}$ blood infusion first followed by $25 \mu \mathrm{L}$ blood infusion 5 min later [26]. The needle was left in place for an additional $5 \mathrm{~min}$ after the completion of $30 \mu \mathrm{L}$ injection to prevent possible leakage, after which was withdrawn slowly at a rate of $1 \mathrm{~mm} / \mathrm{min}$. The cranial burr hole was sealed with bone wax and the incision of scalp was sutured. A $0.4 \mathrm{ml}$ of normal saline was injected subcutaneously to avoid postsurgical dehydration. Mice were allowed to recover fully from anesthesia under close observation. The sham operation was performed following the same procedure without whole blood injection.

\section{Drug Administration}

Met-RANTES (R\&D Systems, Minneapolis, USA) was dissolved in phosphate-buffered saline (PBS). Three different doses of Met-RANTES were tested $(0.15 \mu \mathrm{g} / \mathrm{kg}, 0.5 \mu \mathrm{g} / \mathrm{kg}$, and $1.5 \mu \mathrm{g} / \mathrm{kg}$ ) and were administered intranasally at $1 \mathrm{~h}$ after ICH induction [23]. Ceramide C6 $(5 \mathrm{mg} / \mathrm{kg}$; Santa Cruz Biotechnology, INC., Santa Cruz, USA), a specific ERK1/2 activator, was dissolved in saline and administered intracerebroventricularly at $24 \mathrm{~h}$ before ICH induction [27]. Recombinant CCL5 (rCCL5) (0.5 $\mu \mathrm{g} /$ mouse; R\&D Systems, Minneapolis, USA), dissolved in PBS, was administered via intracerebroventricular in naïve mouse as previously reported [28]. TPR1 CRISPR was used to knockdown TPR1 gene expression in the rat brain. A total of $2 \mu \mathrm{g}$ per pup of TPR1 CRISPR knockout (Santa Cruz Biotechnology, Dallas, TX, MA, USA) or scrambled CRISPR (Santa Cruz Biotechnology, Dallas, TX, USA) was given intracerebroventricularly at a rate of $1 \mu \mathrm{L} /$ min using a pump [29]. 

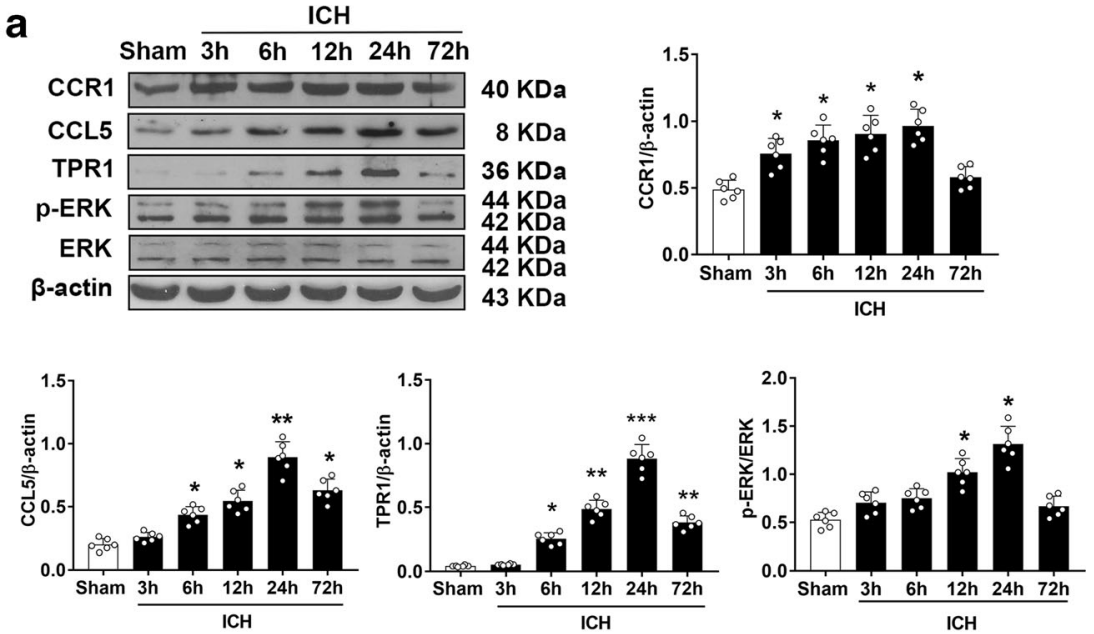

b
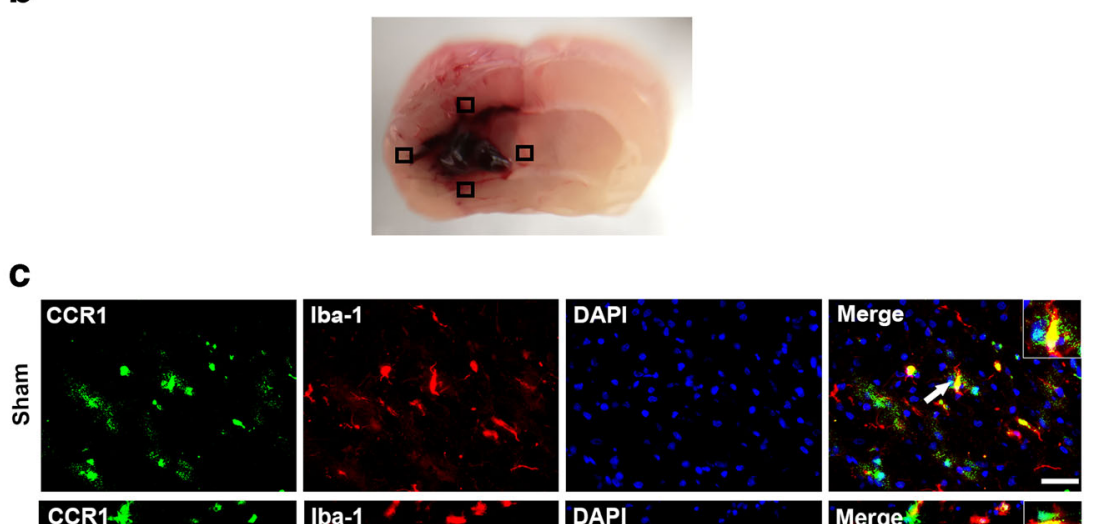

동
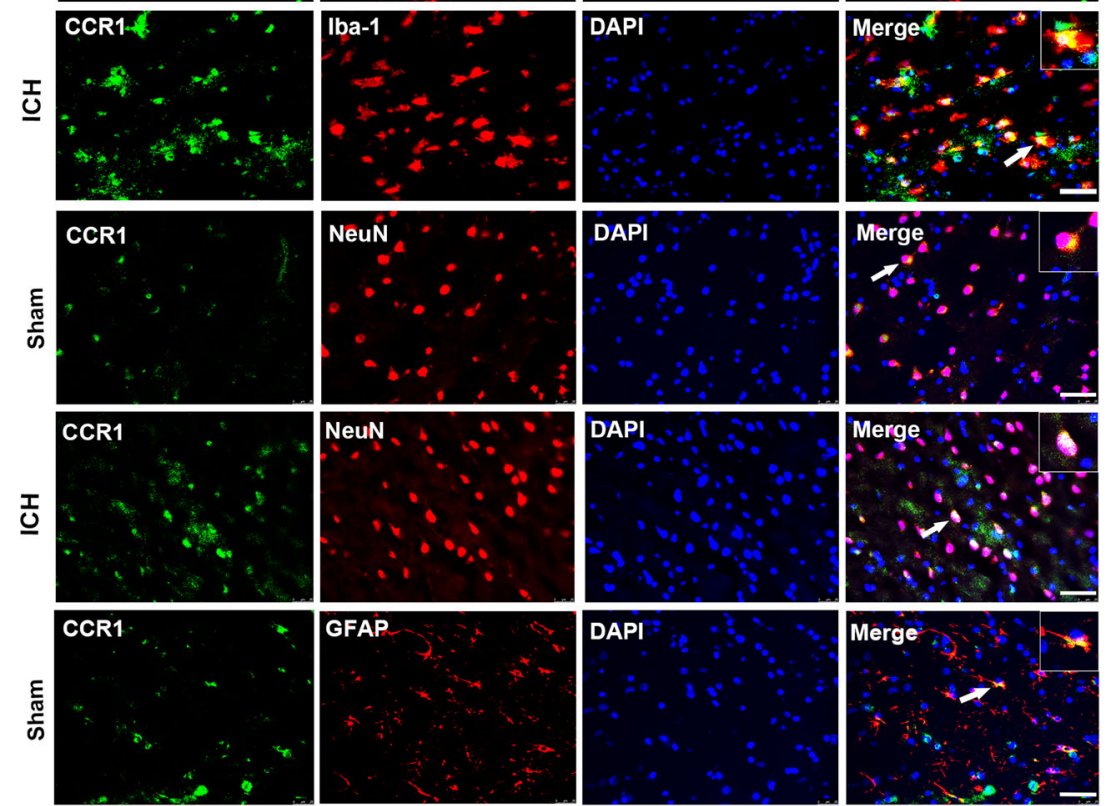

DAPI

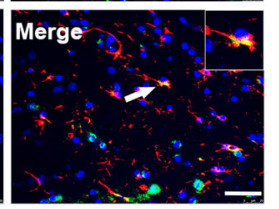

$\underline{\underline{I}}$
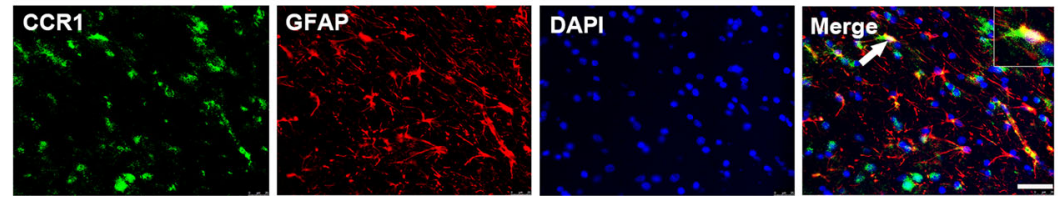
Fig. 1 Time course expression of CCR1, CCL5, TPR1, and p-ERK1/2 after intracerebral hemorrhage (ICH). a Representative Western blot images and quantitative analyses of CCR1, CCL5, TPR1, and p-ERK1/ 2 time course after ICH. Values are expressed as mean \pm SD. $* p<0.05$, ** $p<0.01$ versus sham group. $N=6$. b The schematic illustration of brain tissue shows the four areas of perihematomal region with mark of black squares; the images were taken out for the immunofluorescence staining. c Double immunofluorescence staining for CCR1 (green) in microglia (Iba-1, red), neurons (NeuN, red), and astrocytes (GFAP, red) in the perihematomal area at $24 \mathrm{~h}$ in sham group and ICH $(24 \mathrm{~h})$ group. Scale bar $=50 \mu \mathrm{m} . \quad N=2$. DAPI, 4',6-diamidino-2-phenylindole; Iba-1, ionized calcium-binding adaptor molecule-1; NeuN, neuronal nuclear; GFAP, glial fibrillary acidic protein

\section{Intracerebroventricular Injection}

Intracerebroventricular administration was performed as previously described [30, 31]. Briefly, the 26-gauge needle of a 10 - $\mu$ l Hamilton syringe was inserted into the left lateral ventricle through a cranial burr hole at the following coordinates (0.22 $\mathrm{mm}$ posterior and $1.0 \mathrm{~mm}$ lateral to the bregma, $2.25 \mathrm{~mm}$ deep under dura). A microinfusion pump was used for intracerebroventricular injection at a rate of $0.667 \mu \mathrm{L} / \mathrm{min}$. The needle was left in place for an additional $5 \mathrm{~min}$ at the end of infusion and was removed over a 3-min period. The burr hole was sealed with bone wax.

\section{Short-Term Neurobehavior Assessment}

Short-term neurobehavioral functions were assessed with modified Garcia score test, forelimb placement test, and corner turn test at 24 and $72 \mathrm{~h}$ post-ICH, as previously reported [32]. The Garcia score consisted of seven subtests that evaluated spontaneous activity, axial sensation, vibrissae proprioception, symmetry of limb movement, lateral turning, forelimb walking, and climbing. Each subtest, conducted with a maximum neurological score of 21 , was given a score ranging from 0 to 3 . The forelimb placement test was used to investigate the responsiveness of vibrissae stimulation for mice, and results were recorded as a percentage of the number of successful left forelimb placements out of 10 consecutive stimulations, Then, left forelimb placement was calculated as left forelimb placement/(left forelimb placement + right forelimb placement) $\times 100 \%$. For the corner turn test, animals were arranged to advance into a $30^{\circ}$ corner and exit by turning either to the left or right. Choice of turning was recorded for a total of 10 trials, and a score was calculated as number of left turns/all trials $\times 100$.

\section{Long-Term Neurobehavior Assessment}

Foot fault and rotarod test were performed to assess sensorimotor function, coordination, and balance at 1 ,
2, and 3 week after ICH. Water maze tests (swim distance and escape latency) were performed to evaluate memory and spatial learning at days 21 to 25 after $\mathrm{ICH}$, as previously described [33]. Morris water maze test probe quadrant duration was evaluated at day 25 after ICH.

\section{Brain Water Content Measurement}

Brain water content (BWC) was measured through wet/dry method as previously reported [34]. At 24 or $72 \mathrm{~h}$ after ICH, mice were euthanized by decapitation under deep anesthesia. Brains were removed immediately and divided into five parts: ipsilateral and contralateral cortex, ipsilateral and contralateral basal ganglia, and cerebellum. To obtain the wet weight, every part was immediately weighed through an electric analytic balance (APX-60, Denver Instrument, NY), then dried at $100{ }^{\circ} \mathrm{C}$ for $24 \mathrm{~h}$ to obtain the dry weight. Brain water content was calculated through the following formula: brain water content $(\%)=[($ wet weight - dry weight $) /$ wet weight $] \times$ $100 \%$.

\section{Western Blot Analysis}

At $24 \mathrm{~h}$ after ICH, mice were transcardially perfused with cold PBS under deep anesthesia. Brain samples were quickly extracted and snap frozen in liquid nitrogen. Brain tissues were stored in -80 freezers for western blot. Western blotting was performed as described previously [35]. The ipsilateral/right brain hemispheres were homogenized in RIPA lysis buffer (Santa Cruz Biotechnology, Santa Cruz, CA) and centrifuged at $4{ }^{\circ} \mathrm{C}$ for $30 \mathrm{~min}$ at $14,000 \mathrm{~g}$. Equal amounts of protein were loaded on an SDS-PAGE gel and run using electrophoresis, then transferred to a nitrocellulose membrane. The membrane was blocked and incubated overnight at $4{ }^{\circ} \mathrm{C}$ with the following primary antibody: rabbit anti-CCR1 (1:1000, Thermo Fisher scientific, PA1-41062), mouse anti-CCL5 (1:1000, Santa Cruz sc-373984), rabbit anti-TPR1 (1:1000, Abcam, ab241484), rabbit anti-ERK1/2 (1:10000, Santa Cruz sc292838), rabbit anti-p-ERK1/2 (1:1000, Cell Signaling 9101), rabbit anti-IL-1 $\beta$ (1:1000, Abcam ab9722), rabbit anti-TNF- $\alpha$ (1:1000, Abcam ab6671), mouse anti-MPO (1:1000, Santa Cruz sc-390109), goat anti-Iba-1 (1:1000, Abcam ab5076), mouse anti- $\beta$-actin (1:2000, Santa Cruz sc47778). Beta-actin was used as an internal loading control. The respective secondary antibodies (1:3000, Santa Cruz; 1:5000, Abcam) were incubated for $2 \mathrm{~h}$ at room temperature. The bands were probed with an ECL Plus chemiluminescence reagent Kit (Amersham Biosciences, Arlington Heights, PA) and visualized with the image system (Bio-Rad, Versa Doc, model 4000). Relative density of the protein immunoblot images was analyzed by ImageJ software (ImageJ 1.4, NIH, USA). 


\section{Immunofluorescence Staining}

At $24 \mathrm{~h}$ after ICH, mice were transcardially perfused with cold PBS under deep anesthesia followed with $10 \%$ formalin perfusion. Brains were then removed and fixed in formalin at $4{ }^{\circ} \mathrm{C}$ overnight and dehydrated with $30 \%$ sucrose for 5 days. The frozen coronal slices (10 $\mu \mathrm{m}$ thick) were sectioned in cryostat (CM3050S; Leica Microsystems). Double immunofluorescence staining was performed as previously described [26]. The primary antibodies included rabbit anti-CCR1 (1:200, thermo fisher scientific PA1-41062), anti-Iba-1 (1:200, Abcam ab178847).

\section{Statistical Analysis}

Data were expressed as the mean and standard deviation (mean $\pm \mathrm{SD}$ ). Statistical analysis was performed with Graph Pad Prism (Graph Pad Software, San Diego, CA). Multiple comparisons were statistically analyzed with one-way analysis of variance (ANOVA) followed by Tukey post hoc multiple comparison analysis. Statistical significance was defined as $p<0.05$.
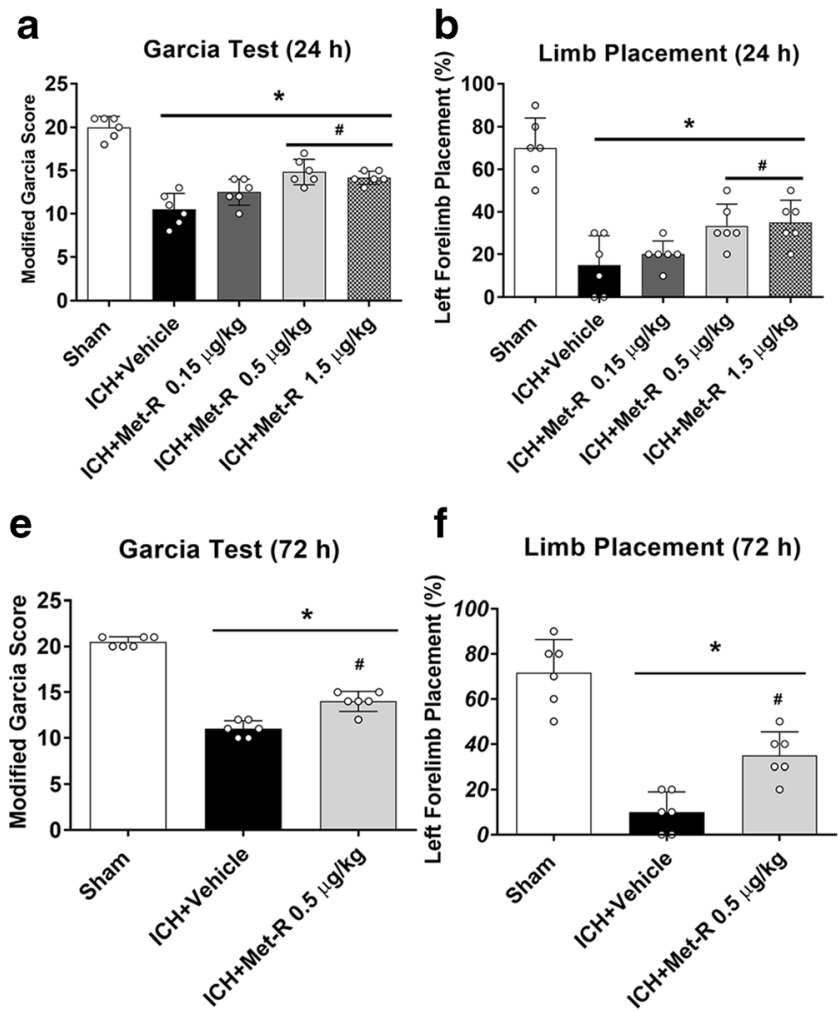

Fig. 2 The effects of different doses of Met-RANTES on neurobehavior tests, brain water content (BWC) at $24 \mathrm{~h}$ and $72 \mathrm{~h}$ post-ICH. a Modified Garcia test, (b) forelimb placement test, (c) corner turn test, and (d) BWC at $24 \mathrm{~h}$ post-ICH. e Modified Garcia test, (f) forelimb placement test, (g) corner turn test, and (h) brain water content at $72 \mathrm{~h}$ after ICH. ${ }^{*} p<0.05$,

\section{Results}

\section{Animal Mortality and Exclusion}

A total of 210 male CD1 mice were used for the study of which 44 were shams, 30 were naïves, and 136 mice underwent ICH induction (Additional File 2: Table S1). None of sham operated mice died. The total mortality rate of ICH was $5.88 \%$ (8/136). The mortality rate was not significantly different among all the ICH experimental groups. None of the mice were excluded from the study (Additional file 2: Table S1).

\section{Time Course and Cellular Expression of CCR1 After ICH}

Western blot was performed to assess the endogenous protein expressions of CCR1, CCL5, TPR1, and p-ERK1/2 in the ipsilateral/right cerebral hemispheres at $3,6,12,24$, and $72 \mathrm{~h}$ after ICH. When compared to the sham group, CCR1 expression started increasing significantly at $3 \mathrm{~h}$ and peaked at $24 \mathrm{~h}$ after ICH. The notable elevation of endogenous CCL5 levels started at $6 \mathrm{~h}$ and peaked at $24 \mathrm{~h}$ after ICH. The expressions of TPR 1 and p-ERK $1 / 2$ showed similar increasing trend after ICH (Fig. 1A). Double immunofluorescence staining revealed that CCR1 was expressed in the microglia, neurons,

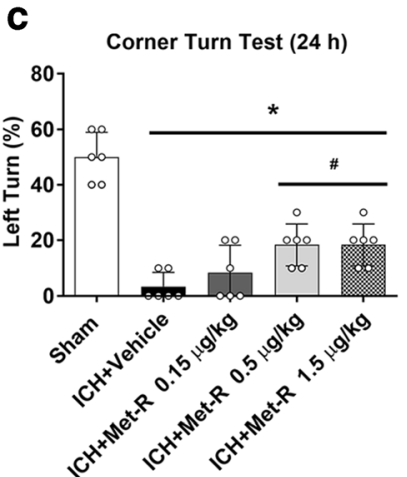

g

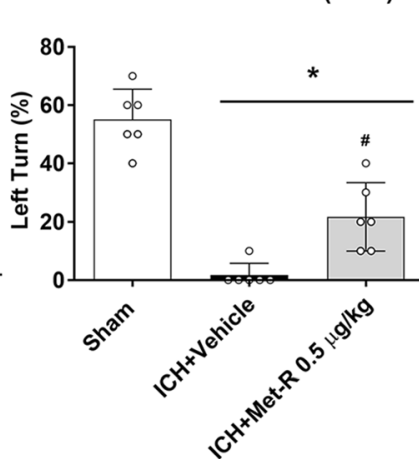

$* * p<0.01$ versus sham group; $\# p<0.05$ versus $\mathrm{ICH}+$ vehicle group. Error bars are represented as mean $\pm \mathrm{SD}$. One-way ANOVA, Tukey's test, $n=6$ per group. Ipsi-BG ipsilateral basal ganglia, Ipsi-CX ipsilateral cortex, Cont-BG contralateral basal ganglia, Cont-CX contralateral cortex, Cerebel cerebellum

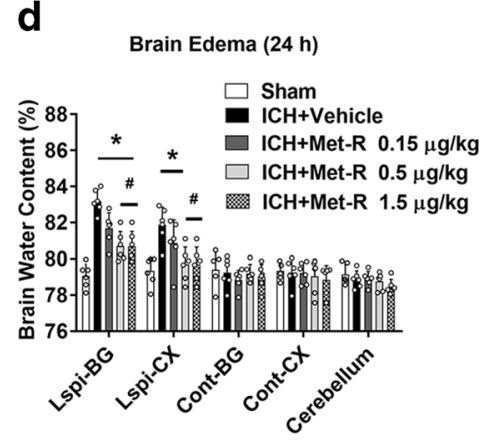

h Brain Edema (72 h)

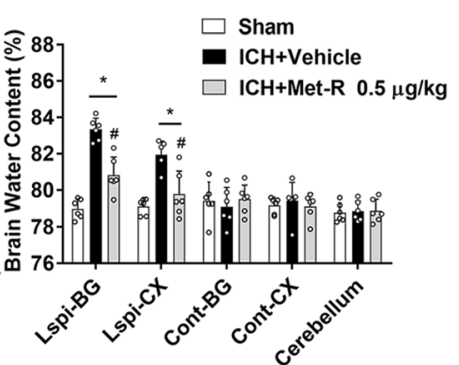


and astrocytes in both shams and ICH mice. Furthermore, there were more CCR1 positive cells visualized within the ipsilateral basal cortex of ICH mice compared to shams (Fig. 1B, C).

\section{CCR1 Inhibition with Met-RANTES Improved Neurobehavioral Deficits and Reduced Brain Edema at $24 \mathrm{H}$ and $\mathbf{7 2} \mathrm{H}$ after ICH}

At $24 \mathrm{~h}$ and $72 \mathrm{~h}$ after ICH, there were significantly lower neurobehavioral score and higher BWC in ICH

Fig. 3 The effects of

Met-RANTES on microglia/ macrophage activation and neutrophil infiltration after ICH. a Representative images of immunofluorescence staining of Iba-1 (red), IL-1 $\beta$ (green), and MPO (green) in the perihematomal area at $24 \mathrm{~h}$ after ICH. The schematic illustration of brain tissue shows the four areas of perihematomal region with mark of black

squares, which was used for Iba1, IL-1 $\beta$, and MPO-positive cell counting in the immunofluorescence staining.

$\mathbf{b}, \mathbf{C}, \mathbf{d}$ Quantitative analyses of Iba-1, IL-1 $\beta$, and MPO-positive cells in the perihematomal area at $24 \mathrm{~h}$ after ICH. e, f, $\mathbf{g}$

Representative Western blot bands and quantitative analyses of Iba-1, IL-1 $\beta$, and MPO protein levels in the ipsilateral hemisphere at $24 \mathrm{~h}$ after ICH.

${ }^{*} p<0.05$ versus sham, $\# p<0.05$ versus $\mathrm{ICH}+$ vehicle. Error bars are represented as mean $\pm \mathrm{SD}$. One-way ANOVA, Tukey's test, $n=6$ per group a

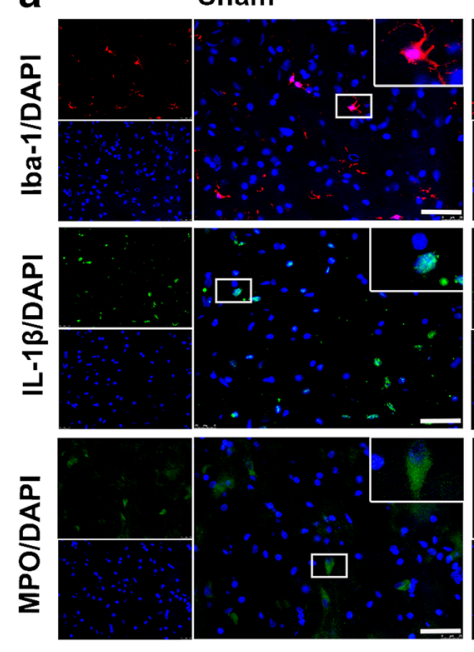

b

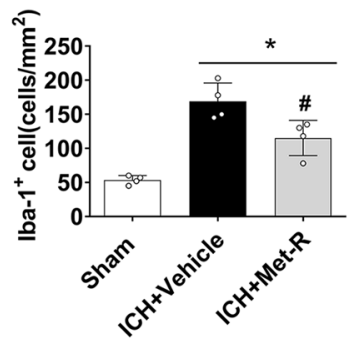

e

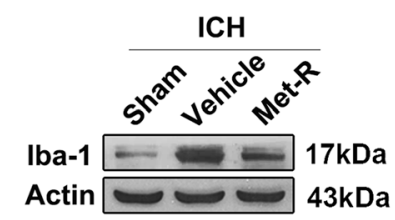

f

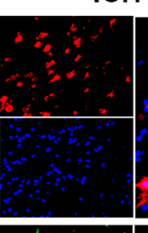

$\mathrm{ICH}+$ Vehicle
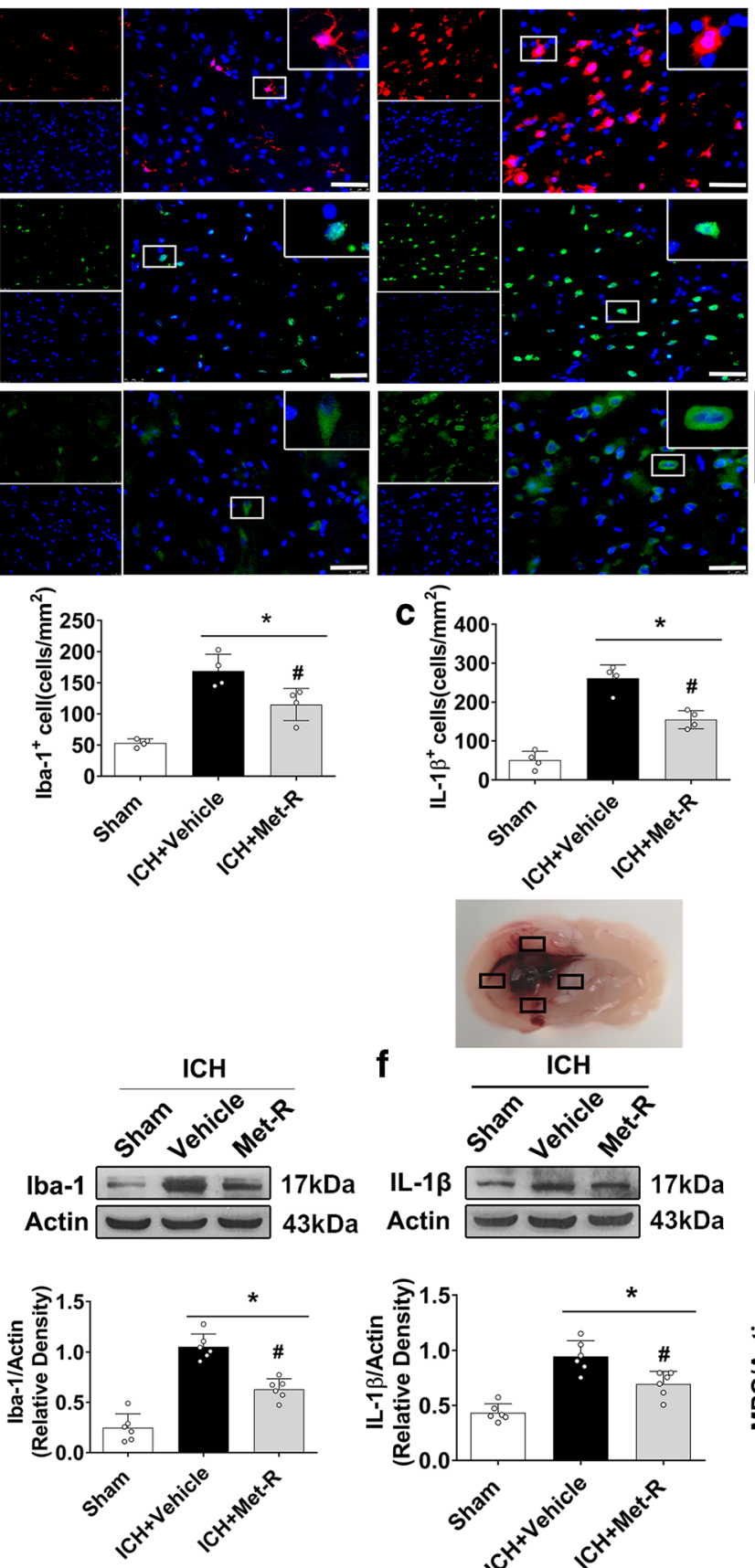

mice than shams. At $24 \mathrm{~h}$ after ICH, Met-RANTES at doses of $0.5 \mu \mathrm{g} / \mathrm{kg}$ and $1.5 \mu \mathrm{g} / \mathrm{kg}$ significantly improved neurological deficits and decreased BWC in the right basal ganglia and cortex when compared to $\mathrm{ICH}+$ vehicle group $(p<0.05$, Fig. $2 \mathrm{~A}-\mathrm{H})$. Based on these results, the dose of $0.5 \mu \mathrm{g} / \mathrm{kg}$ was used as optimal dose in the rest of the experiments involving in MetRANTES treatment. This benefits of Met-RANTES at dose of $0.5 \mu \mathrm{g} / \mathrm{kg}$ on neurobehavioral deficits and BWC persisted to $72 \mathrm{~h}$ after ICH. 


\section{CCR1 Inhibition with Met-RANTES Attenuated Neuroinflammation at 24 H After ICH}

At $24 \mathrm{~h}$ after $\mathrm{ICH}$, there were significantly increased microglia/macrophage activation and neutrophil infiltration compared to shams. Immunofluorescence staining showed that CCR1 inhibition with Met-RANTES significantly reduced the number of Iba-1, IL-1 $\beta$, or MPO-positive cells in the perihematomal area than that in $\mathrm{ICH}+$ vehicle group $(p<0.05$, Fig. 3A-D). Consistently, western blot results showed that the protein levels of Iba-1, IL-1 $\beta$, and MPO in the ipsilateral hemisphere were significantly less in the MetRANTES treated ICH mice when compared to those in the vehicle-treated ICH mice ( $p<0.05$, Fig. $3 \mathrm{E}-\mathrm{G})$.

\section{CCR1 Inhibition with Met-RANTES Improved Long-Term Neurobehavioral Outcomes After ICH}

There were significantly more foot faults of the left forelimb and shortened falling latency in rotarod test in ICH mice compared to shams at days 7, 14, and 21 post-ICH. Met-RANTES treatment significantly improved the behavioral performance in foot fault test and rotarod test in ICH mice $(p<0.05$, Fig. 4A, B). In the Morris water maze, $\mathrm{ICH}+$ vehicle group showed significantly longer swim distance and escape latency compared to the sham group. However, the inhibition of CCR1 with Met-RANTES significantly decreased escape latency and swim distance on blocks 3 to 5 compared to the $\mathrm{ICH}+$ vehicle group $(p<0.05$, Fig. $4 \mathrm{C}, \mathrm{D})$. In the probe quadrant trial, although vehicle-treated ICH mice spent less time in the target quadrant compared with sham group, MetRANTES treatment markedly increased the time spent in the probe quadrant in ICH mice ( $p<0.05$, Fig. 4E, F).

\section{ERK1/2 Activator Reversed the Neuroprotective Effects of Met-RANTES Against Neurobehavioral Deficits and CCR1/TPR1/ERK1/2 Mediated Neuroinflammation at $24 \mathrm{H}$ After ICH}

The pretreatment of ERK1/2 activator ceramide C6 significantly reserved the neurobehavioral benefits of MetRANTES on the modified Garcia test, forelimb placement test, and corner turn test at $24 \mathrm{~h}$ after ICH $(p<0.05$, Fig. $5 \mathrm{~A}, \mathrm{~B}, \mathrm{C})$. In addition, administration of Met-RANTES, ceramide C6, and TPR1 CRISPR did not result in any significant difference in body weight loss compared to the $\mathrm{ICH}+$ vehicle group at $24 \mathrm{~h}$ after ICH $(p<0.05$, Fig. 5D, H).

Met-RANTES significantly reduced protein levels of CCR1, TPR1, p-ERK1/2, and pro-inflammatory cytokine of

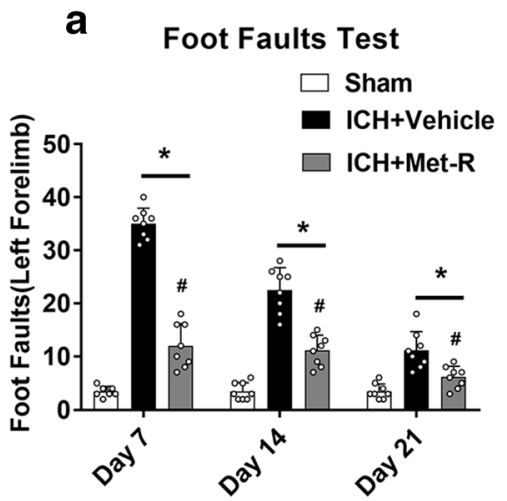

d

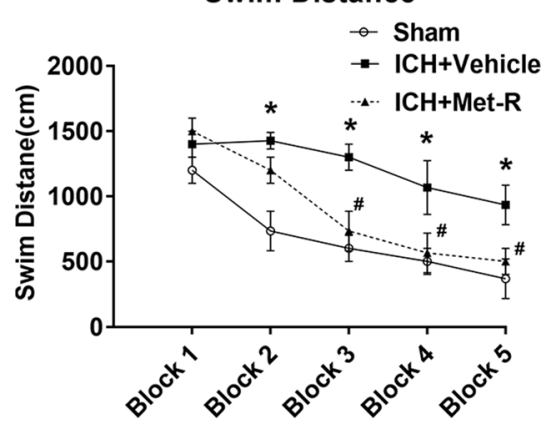

Fig. 4 Met-RANTES improved long-term neurobehavioral function after ICH. a Foot fault test and (b) rotarod test. c Escape latency and (d) swim distance of Morris water maze on 21-25 days after ICH. e Representative heat map in probe test and (f) probe quadrant duration on day 25 post-

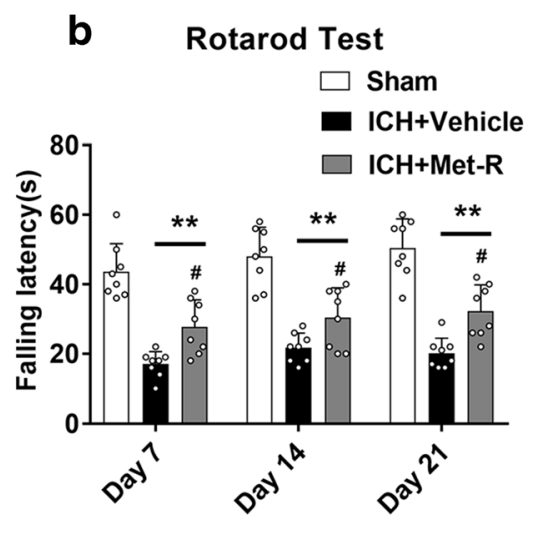

e

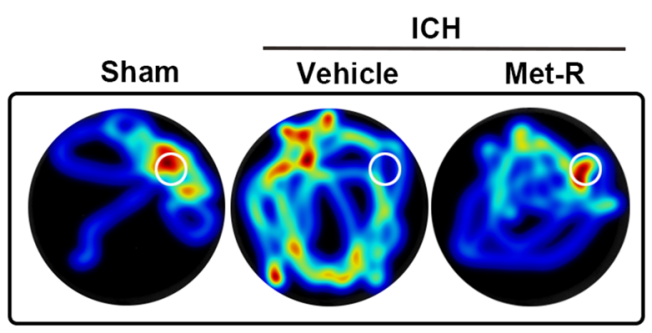

\section{C}

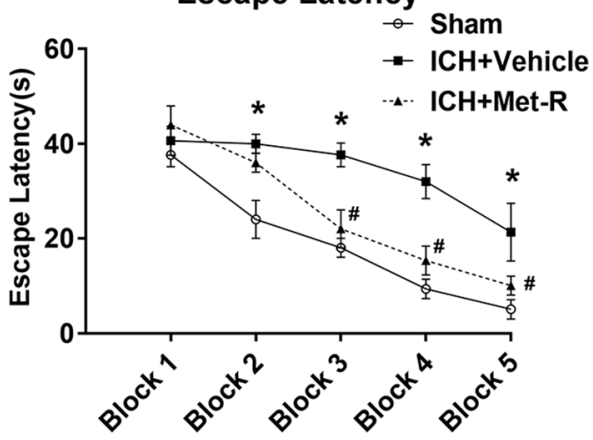

f

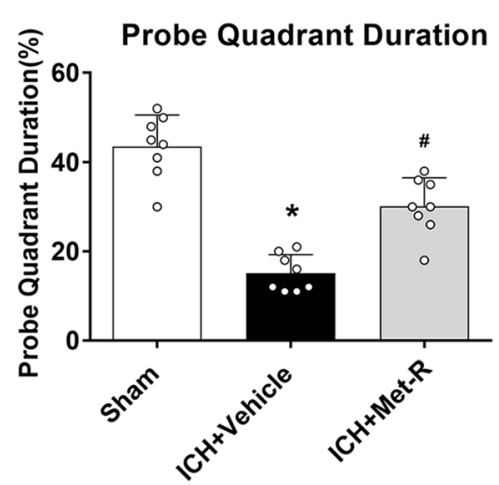

ICH. $* p<0.05, * * p<0.01$ versus sham group; $\# p<0.05$ versus $\mathrm{ICH}+$ vehicle group. Error bars are represented as mean $\pm \mathrm{SD}$. Two-way repeated measures ANOVA, Tukey's test (A-D), and one-way ANOVA, Tukey's test (F), $n=8$ per group 
IL-1 $\beta$ and TNF- $\alpha$ within the ipsilateral hemisphere brain tissues after ICH. Consistently, ceramide C6 reversed effect of Met-RANTES on CCR1/TPR1/ERK1/2 mediated neuroinflammation, which were associated with a higher protein level p-ERK1/2, IL-1 $\beta$, and TNF- $\alpha$ compared to Met-RANTES treated ICH mice (Fig. 6A-F).

\section{CCR1 Activation by rCCL5 Resulted in Neurological Deficits and Neuroinflammation in Naïve Mice Through CCR1/TPR1/ERK1/2 Signaling Pathway at $24 \mathrm{H}$ After rCCL5 Injection}

The intracerebroventricular injection of rCCL5 significantly reduced neurological scores of modified Garcia test, limb placement test, and corner turn test in naïve mice (Fig. 5E, F, G), which were associated with significantly higher protein levels of CCR1, TPR1, and pERK1/2 as well as proinflammatory cytokines of IL- $1 \beta$ and TNF- $\alpha$ in the ipsilateral hemisphere brain tissues. TPR1 CRISPR reversed those effects of rCCL 5 by significantly reduced TPR 1 expressions when compared to Naive + rCCL5 + Scramble CRISPR group $(p<0.05$, Fig. 7A-F).
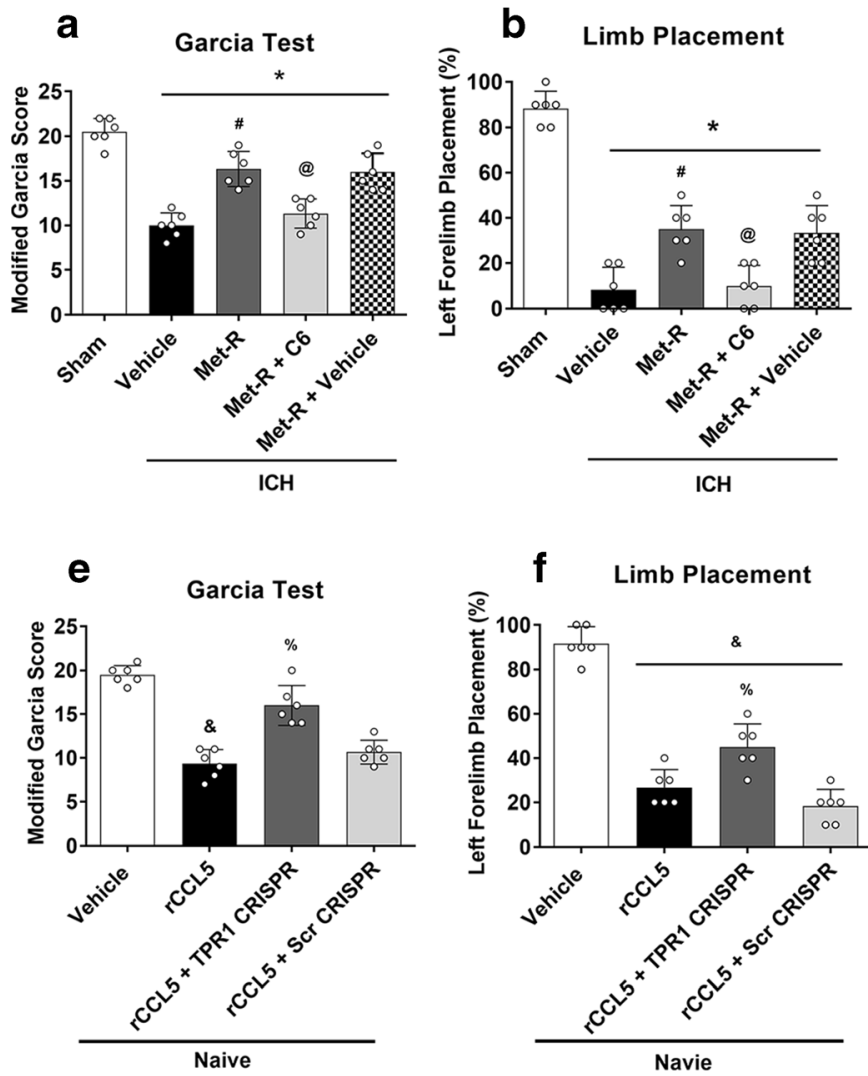

Fig. 5 The ERK1/2 activator reversed effects of Met-RANTES on neurobehavioral outcomes after ICH and TPR1 CRISPR abolished the proinflammatory effects of rCCL5 and improved neurological function. a Modified Garcia test, (b) forelimb placement test, (c) corner turn test, and (d) the effects of Met-RANTES on body weight loss at $24 \mathrm{~h}$ after

\section{Discussion}

In the present study, we investigated the role of CCR1 activation in neuroinflammation after ICH in mice and the possible mechanism involving in CCR1/TPR1/ERK1/2 signaling pathway. We found that 1) the endogenous expressions of CCR1, TPR1, and CCL5 (endogenous ligand of CCR1) were increased within ipsilateral brain hemisphere time-dependently and peaked at $24 \mathrm{~h}$ after ICH. CCR1 were expressed on microglia, neuron, and astrocytes; 2) the intranasal administration of selective CCR1 agonist Met-RANTES $(0.5 \mu \mathrm{g} / \mathrm{kg}$ or $1.5 \mu \mathrm{g} / \mathrm{kg}$ at $1 \mathrm{~h}$ after ICH) significantly improved neurological deficits, reduced brain edema, attenuated microglia/ macrophage activation and neutrophil infiltration, and decreased expressions of CCR1, TPR1, p-ERK1/2, TNF- $\alpha$, and IL- $1 \beta$ at $24 \mathrm{~h}$ after ICH. Conversely, the ERK1/2 activator reversed these effects of Met-RANTES; 3 ) the activation of brain CCR1 with intracerebroventricular administration of rCCL5 resulted in neurologic deficits and neuroinflammation in naïve mice. These detrimental effects in naïve mice were abolished by significantly suppressing brain TPR1 expressions using TPR1 CRISPR. These observations indicated that brain CCR1 activation promoted neuroinflammation after
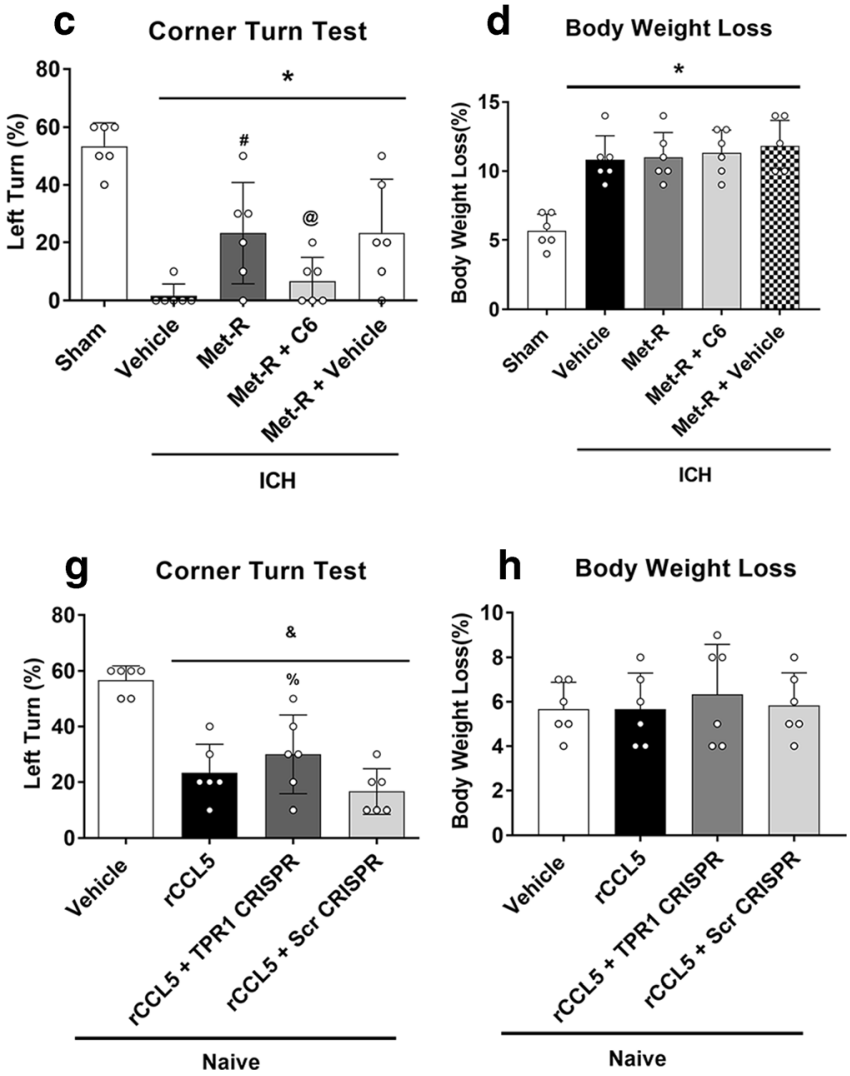

ICH. ${ }^{*} p<0.05$ versus sham, $\# p<0.05$ versus $\mathrm{ICH}+$ vehicle, @ $p<0.05$ versus $\mathrm{ICH}+$ Met $\mathrm{R}+$ Vehicle, and $\& p<0.05$ versus Naive +Vehicle, $\% p<0.05$ versus Naive + rCCL5 + scr CRISPR. Error bars are represented as mean $\pm \mathrm{SD}$. One-way ANOVA, Tukey's test, $n=6$ per group 
ICH, at least partly through CCR1/TPR1/ERK1/2 signaling pathways. The selective CCR1 inhibition with Met-RANTES provided an effective anti-neuroinflammation strategy to improve neurological outcomes in ICH mice.

CCR1 is a membrane receptor widely expressed in the brain, which belongs to chemokines family [36]. Chemokines are a diverse group of small basic proteins that mediate directed migration and activation of target cells [37]. They are classified into four subfamilies, C, CC, CXC, and CX3C, based on structural, functional, and genetic criteria. Previous studies showed that CCR1 was expressed on microglia [10-12]. The CCR1 mRNA and protein levels were increased in brain, spinal cord, peripheral lymphoid organs, and blood plasma at $22 \mathrm{~h}$ after middle cerebral artery occlusion (MCAO) in mice [13]. Consistently, our double immunofluorescence staining results showed that CCR1 expressions were co-localized on microglia and increased after ICH. The increases in brain CCR1 protein level started at an early stage and peaked at $24 \mathrm{~h}$ after ICH, which was accompanied by similar pattern of increases in endogenous ligand CCL5 and TPR1 protein.
Met-RANTES, a selective antagonist for CCR1, was used for the treatment of acute and chronic inflammation [16, 17]. Emerging evidence has demonstrated that Met-RANTES exerts anti-inflammatory effects by inhibiting proinflammatory cytokines production in various diseases including autoimmune encephalomyelitis (EAE) [23], atherosclerosis [38], arthritis [39] and chronic colitis [40]. After ICH, microglial activation and neutrophil infiltration exacerbated the release of pro-inflammatory mediators, such as TNF- $\alpha$, IL- $1 \beta$, and other potentially toxic factors, contributing to brain edema and poor neurological outcomes [7, 41, 42]. Consistent with the previous studies, our results showed that Met-RANTES improved neurobehavioral deficits, decreased brain edema, attenuated microglia/macrophage activation and neutrophil infiltration, and downregulated the expression of proinflammatory cytokines TNF- $\alpha$ and IL- $1 \beta$ in perihematomal areas after ICH.

In this study, we administered Met-RANTES by intranasal route. Intranasal passage can directly deliver various agents to the brain bypassing the blood-brain barrier (BBB). Previous studies showed that neuroprotective agents were delivered to

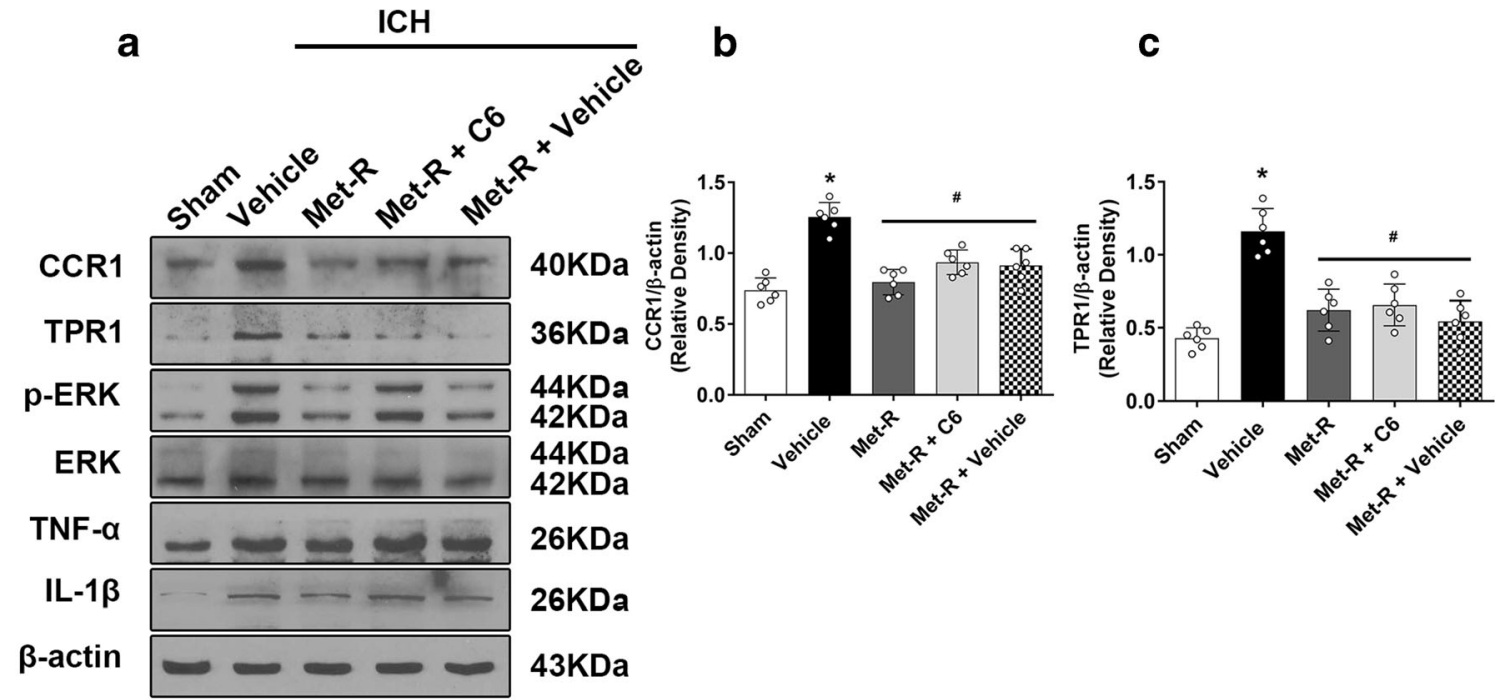

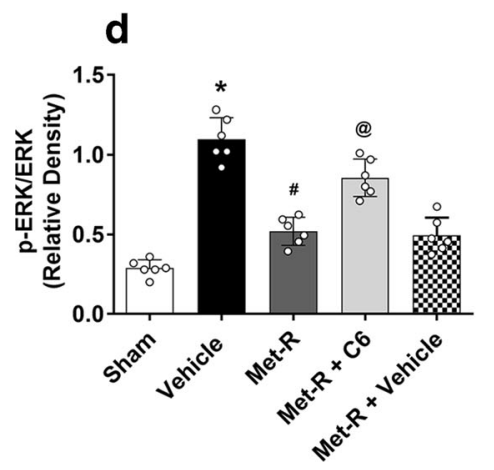

Fig. 6 Activation of ERK1/2 with ceramide C6 abolished the neuroprotective effects of Met-RANTES in ICH mice. a Representative Western blot bands. b-f Quantitative analyses of CCR1, TPR1, p-EPR, TNF- $\alpha$, and IL$1 \beta$ in the ipsilateral hemisphere at $24 \mathrm{~h}$ after ICH. ${ }^{*} p<0.05$ versus sham,
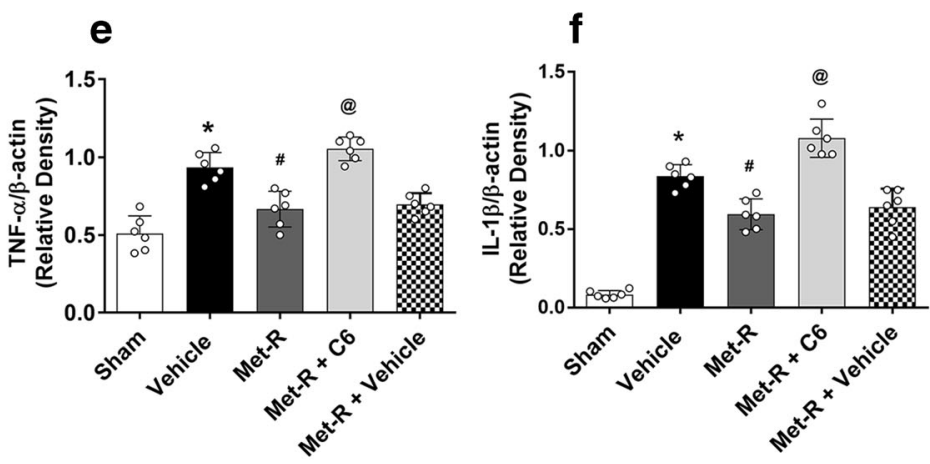

$\# p<0.05$ versus $\mathrm{ICH}+$ vehicle, and @ $p<0.05$ versus $\mathrm{ICH}+$ Met- $\mathrm{R}+$ Vehicle. Error bars are represented as mean $\pm \mathrm{SD}$. One-way ANOVA, Tukey's test, $n=6$ per group 
the brain in preclinical models of stroke [43, 44]. Intranasal method of application is easy and not invasive. Additionally, this is the first study to use Met-RANTES by intranasal route. The inflammatory response after ICH has been reported to initiate $2 \mathrm{~h}$ after ICH and can last up to 5 days [45]. Previous studies showed that Iba- 1 and MPO indicative of microglia/macrophage activation and neutrophil infiltration were detected by immunofluorescence staining and Western blot at $24 \mathrm{~h}$ after ICH $[42,46]$. In this study, mice were treated with Met-RANTES $1 \mathrm{~h}$ after ICH, so that early inflammatory response could be attenuated after ICH to prevent the further progression of injury.

In addition, the early neuroinflammation can cause longterm movement coordination ability, spatial learning, and memory abilities impairment [47]. By attenuating early neuroinflammation, the inhibition of CCR1 with Met-RANTES persistently improved the long-term the sensorimotor and cognitive dysfunction in ICH mice.

Furthermore, we explored the possible mechanism underlying brain CCR1 activation-mediated neuroinflammation after ICH. Previous studies demonstrated the activation of Ras/ MEK/ERK in disease models of cerebral ischemia, oxidative stress, and neurodegenerative disease [48-50]. Ras/MEK/ ERK has been identified as a novel signaling molecule modulating inflammatory response in the arthritis of animal models [51]. A recent study showed that macrophages CCR1 activation promoted the release of TNF- $\alpha$ and IL-1 $\beta$ via activating Ras/MEK/ERK [19]. Additionally, CCR1 stimulation activated ERK and AKT kinases and increase NFKB transcriptional activity in human adipose tissue-derived stromal cells (ADSCs) [22]. Our results demonstrated that CCR1 inhibition with Met-RANTES significantly decreased

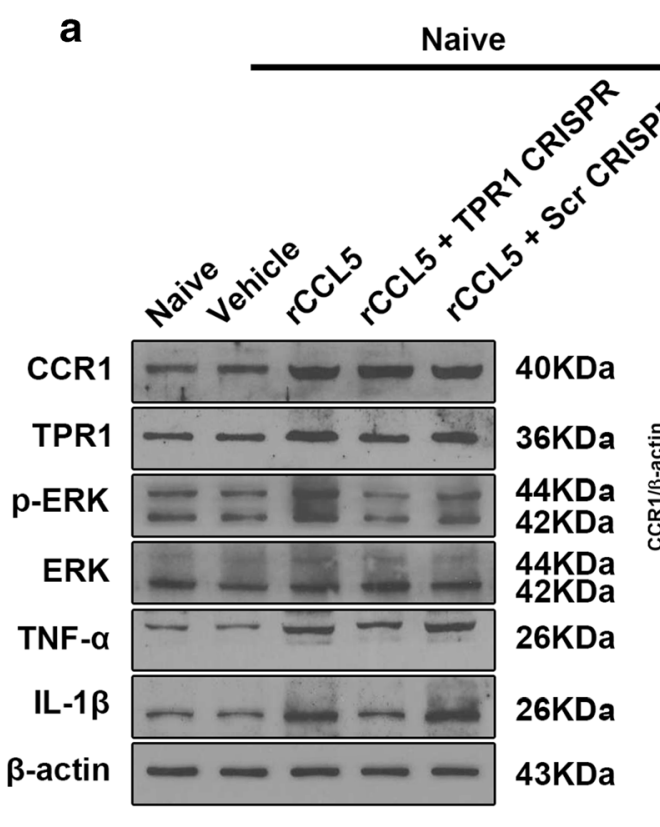

b

C
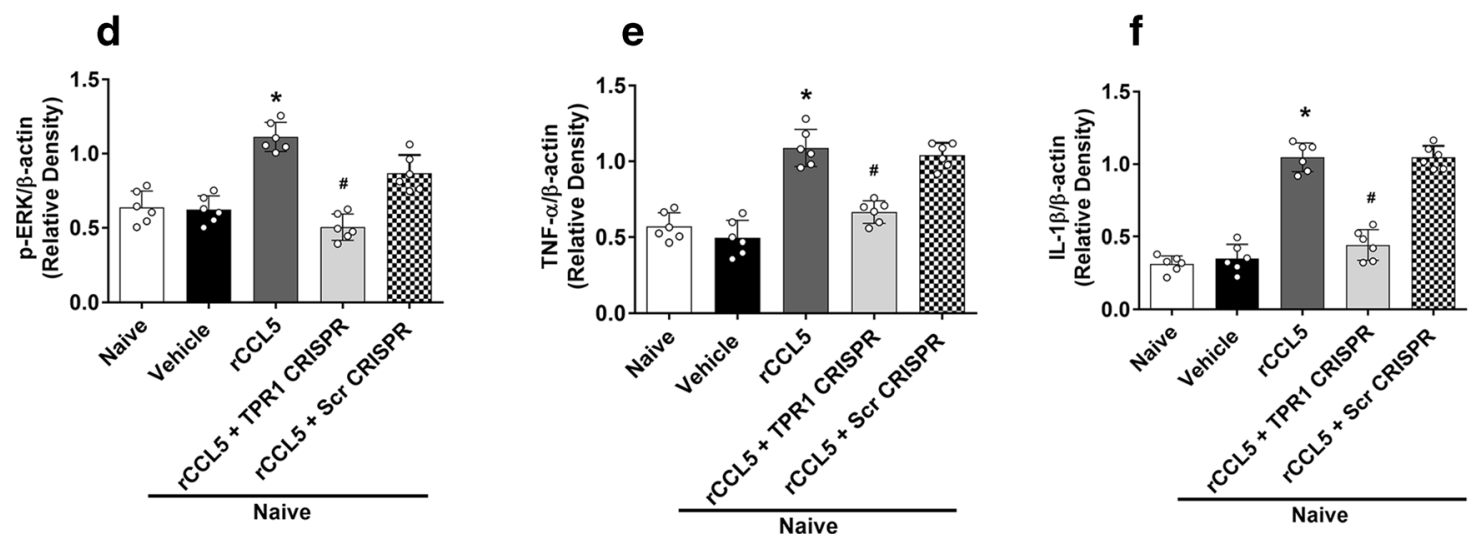

Fig. 7 The effects of rCCL5 and TPR1 CRISPR on the expression of CCR 1 and its downstream signaling proteins in naïve mice. a Representative Western blot bands. b-f Quantitative analyses of CCR1, TPR1, p-ERK1/2, IL-1 $\beta$, and TNF- $\alpha$ in the ipsilateral hemisphere at

$24 \mathrm{~h}$ after rCCL5 administration in naïve mice. ${ }^{*} p<0.05$ versus naive + vehicle and ${ }^{\#} p<0.05$ versus naive + rCCL5 + scr CRISPR. Error bars are represented as mean $\pm \mathrm{SD}$. One-way ANOVA, Tukey's test, $n=6$ per group 
expressions of CCR1 and phosphorylated ERK1/2 as well as downregulated the expressions of pro-inflammatory cytokines including TNF- $\alpha$ and IL-1 $\beta$ at $24 \mathrm{~h}$ after ICH. Ceramide C6, a selective ERK1/2 activator [52] increased the activation of ERK1/2 in cardiovascular diseases. The application of ERK1/2 activator ceramide C6 before ICH induction significantly increased brain tissue p-ERK1/2 and reversed the beneficial effects of Met-RANTES on neurobehavioral deficits and neuroinflammation after ICH. These results suggest that ERK1/2 phosphorylation may be the downstream signaling of CCR1 activationmediated neuroinflammation after ICH.

Interestingly, ceramide 6 did not change the downregulation of brain TPR1 by Met-RANTES. TPR1 is a ubiquitous protein composed of three TPR motifs [18]. Although the biological role of TPR1 has not been well defined at present, it appears to function as a scaffold that TPR1 can link Ga16/ Ga14 activation to Ras signaling in HEK 293 cells [20, 21]. Recent studies have revealed that CCR1 may use the coexistence of Ga14/16 and TPR1 to activate Ras/ERK [19]. We further investigated the role of TPR1 in CCR1 mediated neuroinflammation and its relationship to ERK1/2 activation. Brain CCR1 activation by intracerebroventricular administration of rCCL5 in naïve mice increased the brain expression of CCR1, TPR1, p-ERK1/2, and pro-inflammatory cytokines TNF- $\alpha$ and IL- $1 \beta$ at $24 \mathrm{~h}$ after rCCL5 injection, leading to significant neurological deficits. The detrimental effects of CCR1 activation including ERK1/2 phosphorylation were reversed by TPR1 CRISPR that significantly suppressed brain TPR1 expression, suggesting TPR1 is an upstream signaling of ERK1/2 activation. Also, TPR1 CRISPR did not abolish the rCCL5-induced elevation in protein level of CCR1. Collectively, our findings implicated that CCR1 activation promoted neuroinflammation possibly through CCR1/TPR/ ERK1/2 signaling pathway.

There are some limitations in this study. First, the present study only focused on CCR1 mediated neuroinflammation after ICH. Future studies are needed to explore the role of CCR1 activation in neuronal apoptosis and blood-brain barrier disruption [40, 41]. Second, the pathophysiology of neuroinflammation after ICH is a complex network. We cannot exclude the contribution of Gs/PLC/DAG/PKC signaling in CCR1 mediated inflammation [53]. Third, ICH tends to occur in elderly patient with hypertension and cerebrovascular disease [54]. Further studies are needed to certify the neuroprotection effects of CCR1 inhibition after experimental ICH in different age groups. Also, sexual dimorphism has been shown to exist in neuroinflammation and its mechanisms. Previous studies showed that decreased mortality and improved neurobehavioral outcomes occur in female compared to male mice after ICH $[54,55]$. However, in this study, we used only male mice for our experiments, and the neuroprotective effect of Met-RANTES in ICH female mice model was not evaluated. The lack of sex difference evaluation is a major limitation of the study and needs to be further elucidated in future studies.

\section{Conclusions}

Our study demonstrated that CCR1 activation promoted acute neuroinflammation at least partially via the CCR1/TPR1/ERK pathway in a mouse model of ICH. The inhibition of CCR1 with Met-RANTES improved neurobehavioral deficits, attenuated brain edema, and neuroinflammation. Therefore, targeting CCR1 activation may provide a promising therapeutic strategy in the management of ICH patients.

Required Author Forms Disclosure forms provided by the authors are available with the online version of this article.

Authors' Contributions PS worked on the experimental design. JY, GZ, UO, WNW, SP, and ZDT conducted the experiments, analyzed the data, and drafted the manuscript. PS and LH worked on the manuscript revision. JHZ and JPT participated in the experimental design, data analysis and interpretation, and manuscript preparation. All authors read and approved the final manuscript.

Funding This study was supported by grants from NINDS to JHZ (NS078755) and JT (R21-NS101284).

\section{Compliance with Ethical Standards}

Ethics Approval and Consent to Participate All animal experiments were approved by the Institutional Animal Care and Use Committee at Loma Linda University. The study followed the Health's Guide for the Care and Use of Laboratory Animals (National Research Council) and complied with the ARRIVE guidelines for reporting in vivo experiments.

Competing Interests The authors declare that they have no competing interests.

Abbreviations ARRIVE, animal research: reporting of in vivo experiments; CCR1, C-C chemokine receptor type 1; Cerebel, cerebellum; CNS, central nervous system; Cont-BG, contralateral basal ganglia; Cont-CX, contralateral cortex; GFAP, glial fibrillary acidic protein; Iba1, ionized calcium-binding adaptor molecule; $\mathrm{ICH}$, intracerebral hemorrhage; Ipsi-BG, ipsilateral basal ganglia; Ipsi-CX, ipsilateral cortex; TPR1, tetratricopeptide repeat 1; MPO, myeloperoxidase; Met-R, MetRANTES; NeuN, neuronal specific nuclear protein; PBS, phosphatebuffered saline

\section{References}

1. Keep RF, Hua Y, Xi G. Intracerebral haemorrhage: mechanisms of injury and therapeutic targets. Lancet Neurol 2012;11(8): 720-731.

2. Lapchak PA, Zhang JH. The High Cost of Stroke and Stroke Cytoprotection Research. Transl Stroke Res 2017;8(4): 307-317.

3. Wilkinson DA, Pandey AS, Thompson BG, Keep RF, Hua Y, Xi G. Injury mechanisms in acute intracerebral hemorrhage. Neuropharmacology 2018;134(Pt B):240-248. 
4. Zhou Y, Wang Y, Wang J, Anne Stetler R, Yang QW. Inflammation in intracerebral hemorrhage: from mechanisms to clinical translation. Prog Neurobiol 2014;115: 25-44.

5. Lattanzi S, Brigo F, Trinka E, Cagnetti C, Di Napoli M, Silvestrini M. Neutrophil-to-Lymphocyte Ratio in Acute Cerebral Hemorrhage: a System Review. Transl Stroke Res 2019;10(2): 137-145.

6. Zhang $\mathrm{S}, \mathrm{Hu} \mathrm{ZW}$, Luo HY et al. AAV/BBB-Mediated Gene Transfer of CHIP Attenuates Brain Injury Following Experimental Intracerebral Hemorrhage. Transl Stroke Res 2019.

7. Lu Z, Wang Z, Yu L et al. GCN2 reduces inflammation by peIF2alpha/ATF4 pathway after intracerebral hemorrhage in mice. Exp Neurol 2019;313: 16-25.

8. Li P, Zhao G, Ding Y et al. Rh-IFN-alpha attenuates neuroinflammation and improves neurological function by inhibiting NF-kappaB through JAK1-STAT1/TRAF3 pathway in an experimental GMH rat model. Brain Behav Immun 2019;79:174-185

9. Zhao L, Chen S, Sherchan P et al. Recombinant CTRP9 administration attenuates neuroinflammation via activating adiponectin receptor 1 after intracerebral hemorrhage in mice. J Neuroinflammation 2018;15(1): 215.

10. Sunnemark D, Eltayeb S, Wallstrom E et al. Differential expression of the chemokine receptors CX3CR1 and CCR1 by microglia and macrophages in myelin-oligodendrocyte-glycoprotein-induced experimental autoimmune encephalomyelitis. Brain Pathol 2003;13(4): 617-629.

11. Cowell RM, Xu H, Galasso JM, Silverstein FS. Hypoxic-ischemic injury induces macrophage inflammatory protein-1 alpha expression in immature rat brain. Stroke 2002;33(3): 795-801.

12. Han Y, Wang J, Zhou Z, Ransohoff RM. TGFbetal selectively upregulates CCR 1 expression in primary murine astrocytes. Glia 2000;30(1): 1-10.

13. Offner H, Subramanian S, Parker SM, Afentoulis ME, Vandenbark AA, Hurn PD. Experimental stroke induces massive, rapid activation of the peripheral immune system. J Cereb Blood Flow Metab 2006;26(5): 654-665.

14. Halks-Miller M, Schroeder ML, Haroutunian V et al. CCR1 is an early and specific marker of Alzheimer's disease. Ann Neurol 2003;54(5): 638-646.

15. Proudfoot AE, Buser R, Borlat F et al. Amino-terminally modified RANTES analogues demonstrate differential effects on RANTES receptors. J Biol Chem 1999;274(45): 32478-32485.

16. Liou JT, Mao CC, Ching-Wah Sum D et al. Peritoneal administration of Met-RANTES attenuates inflammatory and nociceptive responses in a murine neuropathic pain model. J Pain : official journal of the American Pain Society 2013;14(1): 24-35.

17. Zhang $X$, Zheng Z, Liu X et al. Tick-borne encephalitis virus induces chemokine RANTES expression via activation of IRF-3 pathway. J Neuroinflammation 2016;13(1): 209.

18. Murthy AE, Bernards A, Church D, Wasmuth J, Gusella JF. Identification and characterization of two novel tetratricopeptide repeat-containing genes. DNA Cell Biol 1996;15(9): 727-735.

19. Lee MM, Chui RK, Tam IY, Lau AH, Wong YH. CCR1-mediated STAT3 tyrosine phosphorylation and CXCL8 expression in THP-1 macrophage-like cells involve pertussis toxin-insensitive Galpha(14/16) signaling and IL-6 release. J Immunol 2012;189(11): 5266-5276.

20. Liu AM, Lo RK, Lee MM et al. Galpha16 activates Ras by forming a complex with tetratricopeptide repeat 1 (TPR1) and Son of Sevenless (SOS). Cell Sign 2010;22(10): 1448-1458.

21. Marty C, Browning DD, Ye RD. Identification of tetratricopeptide repeat 1 as an adaptor protein that interacts with heterotrimeric $\mathrm{G}$ proteins and the small GTPase Ras. Mol Cell Biol 2003;23(11): 3847-3858.
22. Kauts ML, Pihelgas S, Orro K, Neuman T, Piirsoo A. CCL5/CCR1 axis regulates multipotency of human adipose tissue derived stromal cells. Stem Cell Res 2013;10(2): 166-178.

23. Matsui M, Weaver J, Proudfoot AE et al. Treatment of experimental autoimmune encephalomyelitis with the chemokine receptor antagonist Met-RANTES. J Neuroimmunol 2002;128(1-2): 16-22.

24. Rynkowski MA, Kim GH, Komotar RJ et al. A mouse model of intracerebral hemorrhage using autologous blood infusion. Nat Protoc 2008;3(1): 122-128.

25. Zhang P, Wang T, Zhang D et al. Exploration of MST1-Mediated Secondary Brain Injury Induced by Intracerebral Hemorrhage in Rats via Hippo Signaling Pathway. Transl Stroke Res 2019;10(6): 729-743.

26. Rolland WB, Lekic T, Krafft PR et al. Fingolimod reduces cerebral lymphocyte infiltration in experimental models of rodent intracerebral hemorrhage. Exp Neurol 2013;241: 45-55.

27. Zhai L, Sun N, Han Z, Jin HC, Zhang B. Liposomal short-chain C6 ceramide induces potent anti-osteosarcoma activity in vitro and in vivo. Biochem Biophys Res Commun 2015;468(1-2): 274-280.

28. Hang LH, Shao DH, Chen Z, Chen YF, Shu WW, Zhao ZG. Involvement of spinal CC chemokine ligand 5 in the development of bone cancer pain in rats. Basic Clin Pharmacol Toxicol 2013;113(5): 325-328.

29. Gamdzyk M, Doycheva DM, Malaguit J, Enkhjargal B, Tang J, Zhang JH. Role of PPAR-beta/delta/miR-17/TXNIP pathway in neuronal apoptosis after neonatal hypoxic-ischemic injury in rats. Neuropharmacology 2018;140: 150-161.

30. Yu L, Lu Z, Burchell S et al. Adropin preserves the blood-brain barrier through a Notch1/Hes1 pathway after intracerebral hemorrhage in mice. J Neurochem 2017;143(6): 750-760.

31. Wang G, Guo Z, Tong L et al. TLR7 (Toll-Like Receptor 7) Facilitates Heme Scavenging Through the BTK (Bruton Tyrosine Kinase)-CRT (Calreticulin)-LRP1 (Low-Density Lipoprotein Receptor-Related Protein-1)-Hx (Hemopexin) Pathway in Murine Intracerebral Hemorrhage. Stroke 2018;49(12): 3020-3029.

32. Tong LS, Shao AW, Ou YB et al. Recombinant Gas6 augments Axl and facilitates immune restoration in an intracerebral hemorrhage mouse model. J Cereb Blood Flow Metab 2017;37(6): 1971-1981.

33. Lekic T, Hartman R, Rojas $\mathrm{H}$ et al. Protective effect of melatonin upon neuropathology, striatal function, and memory ability after intracerebral hemorrhage in rats. J Neurotrauma 2010;27(3): 627637.

34. Zhao H, Pan P, Yang Y et al. Endogenous hydrogen sulphide attenuates NLRP3 inflammasome-mediated neuroinflammation by suppressing the $\mathrm{P} 2 \mathrm{X} 7$ receptor after intracerebral haemorrhage in rats. J Neuroinflammation 2017;14(1): 163.

35. Pang J, Peng J, Matei N et al. Apolipoprotein E Exerts a WholeBrain Protective Property by Promoting M1? Microglia Quiescence After Experimental Subarachnoid Hemorrhage in Mice. Transl Stroke Res 2018;9(6): 654-668.

36. White GE, Iqbal AJ, Greaves DR. CC chemokine receptors and chronic inflammation-therapeutic opportunities and pharmacological challenges. Pharmacol Rev 2013;65(1): 47-89.

37. Baggiolini M. Chemokines and leukocyte traffic. Nature 1998;392(6676): 565-568.

38. Koenen RR, von Hundelshausen P, Nesmelova IV et al. Disrupting functional interactions between platelet chemokines inhibits atherosclerosis in hyperlipidemic mice. Nat Med 2009;15(1): 97-103.

39. Shahrara S, Proudfoot AE, Woods JM et al. Amelioration of rat adjuvant-induced arthritis by Met-RANTES. Arthritis Rheum 2005;52(6): 1907-1919.

40. Ajuebor MN, Hogaboam CM, Kunkel SL, Proudfoot AE, Wallace JL. The chemokine RANTES is a crucial mediator of the progression from acute to chronic colitis in the rat. J Immunol 2001;166(1): $552-558$. 
41. Gong L, Manaenko A, Fan R et al. Osteopontin attenuates inflammation via JAK2/STAT1 pathway in hyperglycemic rats after intracerebral hemorrhage. Neuropharmacology 2018;138: 160-169.

42. Wang T, Nowrangi D, Yu L et al. Activation of dopamine D1 receptor decreased NLRP3-mediated inflammation in intracerebral hemorrhage mice. J Neuroinflammation 2018;15(1): 2.

43. Topkoru BC, Altay O, Duris K, Krafft PR, Yan J, Zhang JH. Nasal administration of recombinant osteopontin attenuates early brain injury after subarachnoid hemorrhage. Stroke 2013;44(11): 31893194.

44. Doyle KP, Yang T, Lessov NS et al. Nasal administration of osteopontin peptide mimetics confers neuroprotection in stroke. J Cereb Blood Flow Metab 2008;28(6): 1235-1248.

45. Wu H, Zhang Z, Li Y et al. Time course of upregulation of inflammatory mediators in the hemorrhagic brain in rats: correlation with brain edema. Neurochem Int 2010;57(3): 248-253.

46. Chen S, Zhao L, Sherchan $\mathrm{P}$ et al. Activation of melanocortin receptor 4 with RO27-3225 attenuates neuroinflammation through AMPK/JNK/p38 MAPK pathway after intracerebral hemorrhage in mice. J Neuroinflammation 2018;15(1): 106.

47. Zhu W, Gao Y, Chang CF, Wan JR, Zhu SS, Wang J. Mouse models of intracerebral hemorrhage in ventricle, cortex, and hippocampus by injections of autologous blood or collagenase. PLoS One 2014;9(5): e97423.

48. Chen YF, Tsai HY, Wu KJ, Siao LR, Wood WG. Pipoxolan ameliorates cerebral ischemia via inhibition of neuronal apoptosis and intimal hyperplasia through attenuation of VSMC migration and modulation of matrix metalloproteinase-2/9 and Ras/MEK/ERK signaling pathways. PLoS One 2013;8(9): e75654.
49. Arany I, Faisal A, Nagamine Y, Safirstein RL. p66she inhibits prosurvival epidermal growth factor receptor/ERK signaling during severe oxidative stress in mouse renal proximal tubule cells. J Biol Chem 2008;283(10): 6110-6117.

50. Mass E, Jacome-Galarza CE, Blank T et al. A somatic mutation in erythro-myeloid progenitors causes neurodegenerative disease. Nature 2017;549(7672): 389-393.

51. Zayoud M, Marcu-Malina V, Vax E et al. Ras Signaling Inhibitors Attenuate Disease in Adjuvant-Induced Arthritis via Targeting Pathogenic Antigen-Specific Th17-Type Cells. Front Immunol 2017;8: 799 .

52. Vetterkind S, Lin QQ, Morgan KG. A novel mechanism of ERK1/2 regulation in smooth muscle involving acetylation of the ERK1/2 scaffold IQGAP1. Sci Rep 2017;7(1): 9302.

53. Pittaluga A. CCL5-Glutamate Cross-Talk in Astrocyte-Neuron Communication in Multiple Sclerosis. Front Immunol 2017;8: 1079.

54. Xie Y, Li YJ, Lei B et al. Sex Differences in Gene and Protein Expression After Intracerebral Hemorrhage in Mice. Transl Stroke Res 2019;10(2): 231-239.

55. Lei B, Mace B, Bellows ST et al. Interaction between sex and apolipoprotein e genetic background in a murine model of intracerebral hemorrhage. Transl Stroke Res 2012;3(1): 94-101.

Publisher's Note Springer Nature remains neutral with regard to jurisdictional claims in published maps and institutional affiliations. 